\title{
Existence of extended Nash equilibriums of nonmonetized noncooperative games
}

\author{
Chunxiang Zhao, Shihuang Hong* and Chunhong Li
}

"Correspondence:

hongshh@hotmail.com

Department of Mathematics,

Hangzhou Dianzi University, Xuelin

Street, Hangzhou, 310018, China

\section{每 Springer}

\begin{abstract}
In this paper, we consider extended Nash equilibriums of nonmonetized noncooperative games. By using a modified fixed point theorem of set-valued mappings on partially ordered sets, we prove an existence theorem of extended Nash equilibriums of the nonmonetized noncooperative game. Finally, an example is given to illustrate the advantages of our results.
\end{abstract}

MSC: 06A06; 47H10; 58J20; 91A06; 91A10

Keywords: partially ordered set; fixed point; nonmonetized noncooperative game; extended Nash equilibrium

\section{Introduction}

The generalized Nash equilibrium problem is an extension of the standard Nash equilibrium problem, in which the strategy set of each player depends on the strategies of all the other players as well as on his own. This problem has recently attracted much attention due to its applications in various fields like mathematics, management, economics and engineering. For details, we refer the reader to a recent survey paper by Facchinei and Kanzow [1] and the references therein. Very recently, a special class of generalized Nash equilibriums in which the noncooperative games possess strategies as subsets of vector spaces and the ranges of the payoff functions as partially ordered sets (posets) was examined by many researchers, for example, Carl et al. [2], Li et al. [3-5] and Xie et al. [6, 7]. Such games are said to be nonmonetized noncooperative (NNGs for short).

The existence of generalized Nash equilibriums has become the focus of research in NNGs. For instance, in $[2,4]$ the authors proved the existence via different fixed point theorems on Banach lattices. Since a Banach lattice is equipped with some metric topology and algebraic structures, the proofs of the existence follow the same idea as the traditional games, applying fixed point theorems in topological vector spaces. To avoid this, very recently, in [5] the authors obtained the existence results on lattices, which are equipped with neither a topological structure nor an algebraic structure, but only with a special partial order, i.e., a lattice order. Moreover, in [6] the authors extended the concept of generalized Nash equilibrium to extended Nash equilibrium and proved an existence theorem of extended Nash equilibriums of the NNG by applying a fixed point theorem on posets.

Motivated by the works mentioned above, in this paper we also consider extended Nash equilibriums of the NNG and establish an existence result due to an improved fixed point

(c) 2015 Zhao et al.: licensee Springer. This article is distributed under the terms of the Creative Commons Attribution 4.0 International License (http://creativecommons.org/licenses/by/4.0/), which permits unrestricted use, distribution, and reproduction in any medium, provided you give appropriate credit to the original author(s) and the source, provide a link to the Creative Commons license, and indicate if changes were made. 
theorem corresponding to [2] for set-valued mappings on posets without the consideration of a topological structure nor an algebraic structure. We relax the assumptions of the order compactness and the chain-completeness and hence our result compares favorably with that of [6]. Finally, we will give an example to show the advantages of our results.

\section{Preliminaries}

In this section, we recall briefly some definitions and properties of posets. For more details, we refer to [2-6] and [8-10].

Let $\mathcal{P}=(\mathcal{P}, \preceq)$ be a poset with the partial order $\preceq$. An element $u \in \mathcal{P}$ is called an upper bound of a subset $A$ of $\mathcal{P}$ if $x \preceq u$ for each $x \in A$. If $u \in A$, we say that $u$ is the greatest element of $A$ and denote $u=\max A$. The supremum of $A$, denoted by $\sup A$, is an element $u_{0}$ which is an upper bound of $A$ and $u_{0} \preceq v$ as long as $v$ is another upper bound of $A$. It is clear that $\max A=\sup A$ if they exist. The maximal element of $A$ is an element $y \in A$ which satisfies $y=z$ whenever $z \in A$ and $y \preceq z$. Obviously, if $u \in A$ is an upper bound of $A$, then $u$ is a maximal element of $A$. The lower bound, the smallest element $(\min A$ ), the infimum (inf $A$ ), and the minimal element of $A$ can be similarly defined.

A subset $A$ is called a chain if any two elements of $A$ are comparable, i.e., $x \leq y$ or $y \leq x$ for all $x, y \in A$. $A$ is said to be a countable chain if $A$ is a chain and is countable. By c.c. we denote a countable chain. For any $z, w \in \mathcal{P}$, we define the order intervals as follows:

$$
\begin{aligned}
& {[z)=\{x \in \mathcal{P}: z \preceq x\}, \quad(w]=\{x \in \mathcal{P}: x \preceq w\} \quad \text { and }} \\
& {[z, w]=[z) \cap(w]=\{x \in \mathcal{P}: z \preceq x \preceq w\} .}
\end{aligned}
$$

For given posets $\left(X, \preceq^{X}\right)$ and $\left(U, \preceq^{U}\right)$, a single-valued mapping $F$ from $\left(X, \preceq^{X}\right)$ into $\left(U, \preceq^{U}\right)$ is said to be order increasing if $F(x) \preceq^{U} F(y)$ whenever $x \preceq^{X} y$.

In the following definition, we require some weaker conditions compared with Definitions 2.1 and 2.2 of [6].

Definition 2.1 A poset $(\mathcal{P}, \preceq)$ is said to be

(i) inductive if every c.c. $C \subset \mathcal{P}$ has an upper bound in $\mathcal{P}$ and strongly inductive if $\sup C$ exists in $\mathcal{P}$ for every c.c. $C \subset \mathcal{P}$;

(ii) inversely inductive if every c.c. in $\mathcal{P}$ has a lower bound in $\mathcal{P}$ and strongly inversely inductive whenever every c.c. $C \subset \mathcal{P}$ has the infimum in $\mathcal{P}$;

(iii) bi-inductive whenever it is both inductive and inversely inductive and strongly bi-inductive whenever it is both strongly inductive and strongly inversely inductive.

Lemma 2.2 If $\mathcal{P}$ is a bi-inductive poset, then every subset $A \subset \mathcal{P}$ is also a bi-inductive poset.

The following fixed point theorem on posets, which improves the corresponding result of [2], will play an essential role in our main results.

Theorem 2.3 Let $(X, \preceq)$ be a poset. For a given nonempty subset $\mathcal{P}$ of $X$ and a set-valued function $F: X \rightarrow 2^{X}$, we assume that

(H0) there exist $u_{0}, v_{0} \in \mathcal{P}$ with $u_{0} \preceq v_{0}$ such that $F[X]=\bigcup_{x \in X} F(x) \subset\left[u_{0}, v_{0}\right]$;

(H1) if $p \in \mathcal{P}$, then $\min F(p)$ and $\max F(p)$ exist and belong to $\mathcal{P}$. Moreover, $\min F(p)$ is a lower bound of $F[X \cap[p]]$ and $\max F(p)$ is an upper bound of $F[X \cap(p]]$; 
(H2) the set $\{\max F(p): p \in \mathcal{P}\}$ is a strongly inversely inductive subset of $X$ and the set $\{\min F(p): p \in \mathcal{P}\}$ is a strongly inductive subset of $X$.

Then $F$ has the greatest fixed point $u^{*}$ and the smallest fixed point $u_{*}$ in $\mathcal{P}$, that is, $u^{*}=$ $\max \mathscr{F}(X)$ and $u_{*}=\min \mathscr{F}(X)$, where $\mathscr{F}(X)=\{x \in X: x \in F(x)\}$.

Proof Let $G(p)=\min F(p)$ for each $p \in \mathcal{P}$. By virtue of $(\mathrm{H} 1), G$ is well defined and an increasing mapping from $\mathcal{P}$ into itself. From (H2) it follows that every c.c. of $G[\mathcal{P}]$ has a supremum in $\mathcal{P}$. Moreover, $u_{0} \preceq G\left(u_{0}\right)$ with $u_{0}$ given in (H0). Hence, $G\left[\left[u_{0}\right)\right] \subset\left[u_{0}\right)$ by the fact that $G$ is increasing.

In what follows, we prove that $G$ has a fixed point in $\left[u_{0}\right)$. Let $u_{i+1}=G\left(u_{i}\right)$ for $i=0,1, \ldots$. Then $\left\{u_{i}\right\}$ is a c.c. and hence has a supremum by (H2). In addition, $x \preceq G x$ for any $x \in\left\{u_{i}\right\}$. Let $u_{0}^{1}=\sup \left\{u_{i}\right\}$ and $\mathcal{P}_{1}=\left\{u_{i}\right\}_{i=0}^{\infty} \cup\left\{u_{0}^{1}\right\}$. If $u_{0}^{1}=G\left(u_{0}^{1}\right)$, then $G$ has a fixed point. Otherwise, take $u_{i+1}^{1}=G\left(u_{i}^{1}\right)$ for $i=0,1, \ldots$. Again, the set $\left\{u_{0}^{1}, u_{1}^{1}, \ldots\right\}$ has the supremum $u_{0}^{2}=\sup u_{i}^{1}$ by (H2). Denote $\mathcal{P}_{2}=\left\{u_{0}^{1}, u_{1}^{1}, \ldots\right\} \cup\left\{u_{0}^{2}\right\}$. If $u_{0}^{2}=G\left(u_{0}^{2}\right)$, then $G$ has a fixed point. Otherwise, repeating this process, either $G$ has a fixed point, or we can obtain a set sequence $\mathcal{P}_{1}, \mathcal{P}_{2}, \ldots$ satisfying

(i) $\mathcal{P}_{k}=\left\{u_{0}^{k-1}, u_{1}^{k-1}, \ldots\right\} \cup\left\{u_{0}^{k}\right\}$ with $u_{0}^{k}=\sup \left\{u_{i}^{k-1}\right\}$ and $u_{i}^{k}=G\left(u_{i-1}^{k}\right)$ for $i, k=1,2, \ldots$;

(ii) $u_{i-1}^{k} \preceq u_{i}^{k}, u_{j}^{k-1} \preceq u_{t}^{k}$ and $u_{i}^{0}=u_{i}$ for $i, k=1,2, \ldots$ and $j, t=0,1, \ldots$.

Let $\mathcal{Q}=\bigcup_{k=1}^{\infty} \mathcal{P}_{k}$. Then, like the proof of Lemma 2.5 in [11], we can verify that $\mathcal{Q}$ is a c.c., $G(\mathcal{Q}) \subset \mathcal{Q} \subset \mathcal{P}$ and $x \preceq G(x)$ for all $x \in \mathcal{Q}$. (H2) shows that $G$ has the greatest element $u_{*}=\max \mathcal{Q}$. From $G(\mathcal{Q}) \subset \mathcal{Q}$ it follows that $G\left(u_{*}\right) \preceq u_{*}$, while $u_{*} \in \mathcal{Q}$ implies $u_{*} \preceq G\left(u_{*}\right)$. Consequently, $u_{*}$ is a fixed point of $G$ in $\left[u_{0}\right)$ satisfying

$$
u_{*}=\max \mathcal{Q}=\sup G(\mathcal{Q})=\min \left\{u \in\left[u_{0}\right): G(u) \preceq u\right\}
$$

where the last equation is an immediate consequence of Proposition 2.14 in [2].

Now we prove that $u_{*}$ is a lower bound of $\mathscr{F}(X)$. Suppose that this is not true. Then there exists a point $x \in \mathscr{F}(X)$ such that $u_{*} \npreceq x$. Note that $x \in F(x)$, we have $\min \mathcal{Q}=u_{0} \preceq x$ by (H0). On the other hand, for any $y \in \mathcal{Q}$ and $y \preceq x$, in view of the condition (H1) and the definition of $G$, we see that $G(y)$ is a lower bound of $F(x)$. This implies that $G(y) \preceq x$. Now the composition of $\mathcal{Q}$, combining with $u_{0} \in \mathcal{Q}$, guarantees that $u_{*} \preceq x$, which contradicts the choice of $x$. Consequently, $u_{*}$ is a lower bound of $\mathscr{F}(X)$.

Finally, we observe that $u_{*}=G\left(u_{*}\right)=\min F\left(u_{*}\right) \in F\left(u_{*}\right)$, which implies that $u_{*} \in \mathscr{F}(X)$ and hence $u_{*}=\min \mathscr{F}(X)$. Moreover, $u_{*}=\min F\left(u_{*}\right) \in \mathcal{P}$ by $(\mathrm{H} 1)$.

Similar to the above discussion, we can prove the existence of the greatest fixed point $u^{*}$ of $F$. This proof is complete.

\section{Main results}

Definition 3.1 [6] Let $n$ be a positive integer greater than 1. An $n$-person NNG consists of the following elements:

(1) the set of $n$ players, which is denoted by $N=\{1,2, \ldots, n\}$;

(2) the collection of $n$ strategy sets $S=\left\{S_{1}, S_{2}, \ldots, S_{n}\right\}$, where $S_{i}$ stands for the strategy set of player $i$ for $i \in N$, which is also written as $S=S_{1} \times S_{2} \times \cdots \times S_{n}$;

(3) the set of $n$ payoff functions $P=\left\{P_{1}, P_{2}, \ldots, P_{n}\right\}$, where $P_{i}$, a mapping from $S_{1} \times S_{2} \times \cdots \times S_{n}$ into the poset $\left(U, \preceq^{U}\right)$, is the payoff function of player $i$ for $i \in N$;

(4) the outcome space $\left(U, \preceq^{U}\right)$, which is a poset.

This game is denoted by $\Gamma=\{N, S, P, U\}$. 
In an $n$-person NNG $\Gamma=\{N, S, P, U\}$, when all the players simultaneously and independently choose their own strategies $x_{1}, x_{2}, \ldots, x_{n}$ to play, where $x_{i} \in S_{i}$ for $i \in N$, then the player $i$ will receive his payoff $P_{i}\left(x_{1}, x_{2}, \ldots, x_{n}\right) \in U$. For every given $i \in N$ and any $x=\left(x_{1}, x_{2}, \ldots, x_{n}\right) \in S$, we adopt the following notations for convenience:

$$
\begin{aligned}
& -i=N \backslash\{i\}, \quad i=1,2,3, \ldots, n, \\
& x_{-i}=\left(x_{1}, x_{2}, \ldots, x_{i-1}, x_{i+1}, \ldots, x_{n}\right), \\
& S_{-i}=S_{1} \times S_{2} \times \cdots \times S_{i-1} \times S_{i+1} \times \cdots \times S_{n} .
\end{aligned}
$$

Then $x_{-i} \in S_{-i}$, and we can simply write $x$ as $x=\left(x_{i}, x_{-i}\right)$. Moreover, we define $P_{i}\left(S_{i}, x_{-i}\right)=$ $\left\{P_{i}\left(t_{i}, x_{-i}\right): t_{i} \in S_{i}\right\}$.

Definition 3.2 [6] In an $n$-person NNG $\Gamma=\{N, S, P, U\}$, a selection of strategies $\tilde{x}=$ $\left(\tilde{x}_{1}, \tilde{x}_{2}, \ldots, \tilde{x}_{n}\right) \in S_{1} \times S_{2} \times \cdots \times S_{n}$ is called an extended Nash equilibrium of this game if the order relation

$$
P_{i}\left(\tilde{x}_{i}, \tilde{x}_{-i}\right) \nprec^{U} P_{i}\left(x_{i}, \tilde{x}_{-i}\right), \quad \forall x_{i} \in S_{i},
$$

holds for every $i \in N$.

Lemma 3.3 [6] Let $\left(S_{i}, \preceq_{i}\right)$ be a poset for every $i \in N$. Let $S=S_{1} \times S_{2} \times \cdots \times S_{n}$ be the Cartesian product space of $S_{1}, S_{2}, \ldots, S_{n}$, and let $\preceq^{S}$ be the coordinate partial order on $S$ induced by the partial order $\preceq_{i}$, that is, for any $x, y \in S$ with $x=\left(x_{1}, x_{2}, \ldots, x_{n}\right)$ and $y=$ $\left(y_{1}, y_{2}, \ldots, y_{n}\right)$, we have

$$
\begin{aligned}
& x \preceq^{S} y \quad \text { if and only if } \quad x_{i} \preceq_{i} y_{i}, \quad \forall i \in N \quad \text { and } \\
& x \prec^{S} y \quad \text { if and only if } \quad x_{i} \preceq_{i} y_{i} \text { and } x_{i_{0}} \neq y_{i_{0}} \quad \text { for some } i_{0} \in N .
\end{aligned}
$$

Then $\left(S, \preceq^{S}\right)$ is a poset. Furthermore, if every $\left(S_{i}, \preceq_{i}\right)$ is (strongly) inductive, then $\left(S, \preceq^{S}\right)$ is also (strongly) inductive. If every $\left(S_{i}, \preceq_{i}\right)$ is (strongly) bi-inductive, then $\left(S, \preceq^{S}\right)$ is also (strongly) bi-inductive.

Let $\mathscr{P}=\{A: A \subset S$ is a c.c. $\}$. We introduce a partial order on $\mathscr{P}$ as follows: $A \preceq B$ if and only if $A \subseteq B$ and $A \prec B$ if and only if $A \preceq B$ but $A \neq B$ for all $A, B \in \mathscr{P}$.

Lemma 3.4 $(\mathscr{P}, \preceq)$ has a maximal element and a minimal element.

Proof On the contrary, suppose that, for each $A \in \mathscr{P}$, there exists at least an element $B \in \mathscr{P}$ such that $A \prec B$. Let $f(A)=B$. Then $f$ is a mapping from $\mathscr{P}$ into itself and satisfies $A \prec f(A)$ for each $A \in \mathscr{P}$. We assert that every countable chain of $\mathscr{P}$ has a supremum in $\mathscr{P}$. In fact, if $\mathscr{C}=\left\{A_{1}, A_{2}, \ldots\right\}$ is a countable chain of $\mathscr{P}$, then $A_{k}$ is a countable subset of $S$ for $k=1,2, \ldots$ Let $A=\bigcup_{k=1}^{\infty} A_{k}$. It is easy to see that $A$ belongs to $\mathscr{P}$ and is a supremum of $\mathscr{C}$. In the light of Lemma 2.5 in [11], $f$ has a fixed point $A_{0}=f\left(A_{0}\right) \in \mathscr{P}$. On the other hand, from the definition of $f$ we have $A_{0} \prec f\left(A_{0}\right)$. This is a contradiction. Therefore, $\mathscr{P}$ has a maximal element. Analogously, we can prove the existence of a minimal element. This proof is complete. 
In this sequel, the maximal element (resp. minimal element) of $\mathscr{P}$ is said to be a maximal c.c. (resp. minimal c.c.) of $S$.

Lemma 3.5 If $S$ is inductive (inversely inductive), then $S$ has a maximal element $s^{0}$ (minimal element $\left.s_{0}\right)$. Moreover, $\sup \mathcal{P}^{*}$ exists and equals $s^{0}\left(\inf \mathcal{P}_{*}\right.$ exists and equals $\left.s_{0}\right)$ where $\mathcal{P}^{*}\left(\mathcal{P}_{*}\right)$ is a maximal c.c. (minimal c.c.) of $S$.

Proof Lemma 3.4 guarantees the existence of the maximal c.c. $\mathcal{P}^{*}$ of $S$ and the inductive hypothesis further guarantees the existence of the upper bound $s^{0}$ of $\mathcal{P}^{*}$. We first check that $s^{0}$ is a maximal element of $S$. To this end, we choose an element $x \in S$ with $s^{0} \preceq^{S} x$. If $x \notin \mathcal{P}^{*}$, then $B=\mathcal{P}^{*} \cup\{x\}$ is also a c.c., i.e., $B \in \mathscr{P}$. Obviously, $\mathcal{P}^{*} \prec B$, which is a contradiction since $\mathcal{P}^{*}$ is the maximal element of $\mathscr{P}$. Consequently, $x \in \mathcal{P}^{*}$. Since $s^{0}$ is the upper bound of $\mathcal{P}^{*}$, we have $x \preceq^{s} s^{0}$. Hence $x=s^{0}$ and this implies that $s^{0}$ is a maximal element of $S$. Note that $s^{0} \in \mathcal{P}^{*}$, we see that $s^{0}$ is the supremum of $\mathcal{P}^{*}$. The proof for the existence of a minimal element of $S$ is analogous. This proof is complete.

We are in a position to state and prove the main result of this paper.

Theorem 3.6 Let $\Gamma=\{N, S, P, U\}$ be an n-person NNG. If the following conditions hold:

(I) every player's strategy set $\left(S_{i}, \preceq_{i}\right)(i \in N)$ is a strongly inductive and inversely inductive poset,

(II) every player's payoff function $P_{i}: S \rightarrow U(i \in N)$ satisfies

$$
P_{i}(x) \preceq^{U} P_{i}(y) \quad \text { if and only if } x \preceq^{S} y \text { for any } x, y \in S \text { and } i \in N \text {, }
$$

then the game $\Gamma$ has an extended Nash equilibrium. Furthermore, $\Gamma$ has minimal and maximal extended Nash equilibriums.

Proof Lemma 3.4 guarantees that $S$ has at least a maximal c.c. $\mathcal{P}$. Let $\mathcal{P}=\left\{\xi_{1}, \xi_{2}, \ldots\right\}$, where $\xi_{k}=\left(x_{1}^{k}, x_{2}^{k}, \ldots, x_{n}^{k}\right)$ and $\xi_{j}=\left(x_{1}^{j}, x_{2}^{j}, \ldots, x_{n}^{j}\right)$ are comparable with respect to $\preceq^{S}$ for $k, j=1,2, \ldots$. For any fixed $i \in N$, let $S_{i}^{0}=\left\{x_{i}^{k}\right\}_{k=1}^{\infty}$. Then $S_{i}^{0} \subset S_{i}$ and $S_{i}^{0}$ is obviously a c.c. of $S_{i}$ for $i \in N$. Hence there exist an upper bound $\tilde{s}_{i}$ and a lower bound $\tilde{t}_{i}$ of $S_{i}^{0}$ since $S_{i}$ is bi-inductive for $i \in N$. We will verify that $S_{i}^{0}$ is a maximal c.c. of $S_{i}$ for $i \in N$. Suppose that this is not true. There exists some $i_{0} \in N$ such that $S_{i_{0}}^{0}$ is not a maximal c.c. of $S_{i_{0}}$,i.e., there exists another c.c. $A_{i_{0}}$ of $S_{i_{0}}$ such that $S_{i_{0}}^{0} \subset A_{i_{0}}$ and hence there exists $a$ with $a \in A_{i_{0}}$ and $a \notin S_{i_{0}}^{0}$. We have three cases:

Case 1. $a \prec_{i_{0}} x_{i_{0}}^{k}$ for all $k=1,2, \ldots$;

Case 2. $x_{i_{0}}^{k} \prec_{i_{0}}$ a for all $k=1,2, \ldots$;

Case 3. there exists a positive integer $k_{1}$ such that $x_{i_{0}}^{k_{1}} \prec_{i_{0}} a \prec_{i_{0}} x_{i_{0}}^{k_{1}+1}$.

In case 1 , let $\eta=\left(y^{1}, y^{2}, \ldots, y^{n}\right)$ with $y^{i}=\tilde{t}_{i}$ if $i \neq i_{0}$ and $y^{i_{0}}=a$. Then $\eta \notin \mathcal{P}$ and $\eta \prec^{S} \xi_{k}$ for $k=1,2, \ldots$ Thus $\{\eta\} \cup \mathcal{P}$ is a c.c. of $S$ and $\mathcal{P} \subset\{\eta\} \cup \mathcal{P}$, which contradicts the maximality of $\mathcal{P}$. Assume that case 2 occurs. We take $y^{i}=\tilde{s}_{i}$ instead of $y^{i}=\tilde{t}_{i}$ if $i \neq i_{0}$ and $y^{i_{0}}=a$ for every component $y^{i}$ of $\eta$ given in case 1 . Thus we have that $\eta \notin \mathcal{P}, \xi_{k} \prec^{S} \eta, \mathcal{P} \subset\{\eta\} \cup \mathcal{P}$ and $\{\eta\} \cup \mathcal{P}$ is a c.c. of $S$, which contradicts the maximality of $\mathcal{P}$ again. If case 3 occurs, then, for $\eta$ given in case 1 , instead of $y^{i}=\tilde{t}_{i}$, we choose $y^{i}=x_{i}^{k_{1}}$ for $i \neq i_{0}$ and $y^{i_{0}}=a$. Hence $\xi_{k_{1}} \prec^{S} \eta \prec^{S} \xi_{k_{1}+1}$. It is similarly able to get a contradiction. Consequently, $S_{i}^{0}$ is a maximal c.c. of $S_{i}$ for $i \in N$. 
From Lemma 3.5 it follows that the supremum $s_{i}^{0}$ and the infimum $s_{0 i}$ of $S_{i}^{0}$ exist for $i \in N$. We next claim that $s_{i}^{0}=\max S_{i}^{0}$ and $s_{0 i}=\min S_{i}^{0}$. In fact, if $s_{i}^{0} \neq \max S_{i}^{0}$, then $s_{i}^{0} \notin S_{i}^{0}$ and $S_{i}^{0} \subset S_{i}^{0} \cup\left\{s_{i}^{0}\right\}$ is a c.c. of $S_{i}$, which contradicts the maximality of $S_{i}^{0}$. Analogously, we can prove $s_{0 i}=\min S_{i}^{0}$. Let $\xi^{0}:=\left(s_{1}^{0}, s_{2}^{0}, \ldots, s_{n}^{0}\right)$ and $\xi_{0}:=\left(s_{01}, s_{02}, \ldots, s_{0 n}\right)$. It is clear that $\xi^{0}, \xi_{0} \in \mathcal{P}$. This further implies that $\xi^{0}=\max \mathcal{P}$ and $\xi_{0}=\min \mathcal{P}$. In addition, it is easy to see that, for any $x_{-i} \in S_{-i}, P_{i}\left(s_{i}^{0}, x_{-i}\right)$ (resp. $\left.P_{i}\left(s_{0 i}, x_{-i}\right)\right)$ is a maximal element (resp. minimal element) of $P_{i}\left(S_{i}, x_{-i}\right)$ with respect to the partial order $\preceq^{U}$.

For every fixed $i \in N$, we define a set-valued mapping $T_{i}: S \rightarrow 2^{S_{i}} \backslash\{\varnothing\}$ by

$$
T_{i}(x)=\left\{z_{i} \in S_{i} \cap\left[s_{0 i}, s_{i}^{0}\right]: P_{i}\left(z_{i}, x_{-i}\right) \text { is a maximal element of } P_{i}\left(S_{i}, x_{-i}\right)\right\}
$$

for all $x=\left(x_{1}, x_{2}, \ldots, x_{n}\right) \in S$. We have revealed $s_{i}^{0} \in T_{i}(x)$, which illustrates that $T_{i}(x)$ is nonempty for every $x \in S$. Apparently, $\min T_{i}(x)$ and $\max T_{i}(x)$ exist in $\left[s_{0 i}, s_{i}^{0}\right]$ for every $x \in S$, moreover, $\max T_{i}(x)=s_{i}^{0}$. Let us define the set-valued mapping $T: S \rightarrow 2^{S} \backslash\{\varnothing\}$ as follows: for any $x \in S$

$$
T(x)=\left\{y=\left(y_{1}, y_{2}, \ldots, y_{n}\right): y_{i} \in T_{i}(x), i \in N\right\} .
$$

From the definition of $T$, it follows that $\max T(x)=\xi^{0}$ and $\min T(x)=\left(\min T_{1}(x)\right.$, $\left.\min T_{2}(x), \ldots, \min T_{n}(x)\right)$ for every $x \in S$. This implies that $T(S) \subseteq\left[\xi_{0}, \xi^{0}\right]$ and hence $T$ satisfies the hypothesis (H0) of Theorem 2.3.

For any $\xi_{k}=\left(x_{1}^{k}, x_{2}^{k}, \ldots, x_{n}^{k}\right) \in \mathcal{P}$, we observe that $\max T\left(\xi_{k}\right)=\xi^{0}=\left(s_{1}^{0}, s_{2}^{0}, \ldots, s_{n}^{0}\right)$ and $\min T\left(\xi_{k}\right)=\left(z_{1}^{\prime}, z_{2}^{\prime}, \ldots, z_{n}^{\prime}\right)$ with $z_{i}^{\prime}=\min T_{i}\left(\xi_{k}\right)(i \in N)$. Let $z^{\prime}=\left(z_{1}^{\prime}, z_{2}^{\prime}, \ldots, z_{n}^{\prime}\right)$. Then $\min T\left(\xi_{k}\right)=z^{\prime}$. Taking any $x^{\prime \prime} \in S \cap\left(\xi_{k}\right]$ and $z_{i}^{\prime \prime} \in T_{i}\left(x^{\prime \prime}\right)$ for $i \in N$, we have $z_{i}^{\prime \prime} \preceq_{i} s_{i}^{0}=$ $\max T_{i}\left(\xi_{k}\right)$, i.e., $\max T_{i}\left(\xi_{k}\right)$ is an upper bound of $T_{i}\left(x^{\prime \prime}\right)$. The arbitrariness of $x^{\prime \prime}$ induces that $\max T_{i}\left(\xi_{k}\right)$ is also an upper bound of $T_{i}\left[S \cap\left(\xi_{k}\right]\right]$ and the arbitrariness of $i \in N$ induces that $\max T\left(\xi_{k}\right)$ is an upper bound of $T\left[S \cap\left(\xi_{k}\right]\right]$. Similarly, taking any $y^{\prime \prime} \in S \cap\left[\xi_{k}\right)$, by the condition (II), we get $P_{i}\left(z_{i}^{\prime}, x_{-i}^{k}\right) \preceq^{U} P_{i}\left(z_{i}^{\prime}, y_{-i}^{\prime \prime}\right) \preceq^{U} P_{i}\left(z_{i}^{\prime \prime}, y_{-i}^{\prime \prime}\right)$, which implies that $\min T_{i}\left(\xi_{k}\right)=z_{i}^{\prime} \preceq_{i} z_{i}^{\prime \prime}$. Hence $\min T_{i}\left(\xi_{k}\right)$ is a lower bound of $T_{i}\left(y^{\prime \prime}\right)$. The arbitrariness of $y^{\prime \prime}$ guarantees that $\min T_{i}\left(\xi_{k}\right)$ is a lower bound of $T_{i}\left[S \cap\left[\xi_{k}\right)\right]$ and the arbitrariness of $i \in N$ reduces that $\min T\left(\xi_{k}\right)$ is a lower bound of $T\left[S \cap\left[\xi_{k}\right)\right]$ once more. Consequently, $T$ satisfies (H1) of Theorem 2.3.

Note that $\left\{\max T\left(\xi_{k}\right): \xi_{k} \in \mathcal{P}\right\}=\left\{\xi^{0}\right\}$, Obviously, it is a strongly inversely inductive subset of $S$. Since $S_{i}$ is a strongly inductive poset, Lemma 3.3 induces that $S$ is also a strongly inductive poset. In the light of Lemma 2.2, the set $\left\{\min T\left(\xi_{k}\right): \xi_{k} \in \mathcal{P}\right\} \subseteq S$ is a strongly inductive subset of $S$. Therefore, $T$ satisfies (H2) of Theorem 2.3. As a conclusion of Theorem 2.3, $T$ has the greatest fixed point $x^{*}$ and the smallest fixed point $x_{*}$ in $\mathcal{P}$.

Suppose that $\tilde{x}$ is a fixed point of $T$, that is, $\tilde{x}_{i} \in T_{i}(\tilde{x})$, which yields, for every fixed $i \in N$, $P_{i}\left(\tilde{x}_{i}, \tilde{x}_{-i}\right)$ is a maximal element of $P_{i}\left(S_{i}, \tilde{x}_{-i}\right)$. It is equivalent to

$$
P_{i}\left(\tilde{x}_{i}, \tilde{x}_{-i}\right) \nprec^{U} P_{i}\left(t_{i}, \tilde{x}_{-i}\right), \quad \forall t_{i} \in S_{i}
$$

for every $i \in N$, which indicates that $\tilde{x}=\left(\tilde{x}_{1}, \tilde{x}_{2}, \ldots, \tilde{x}_{n}\right)$ is an extended Nash equilibrium. Moreover, from Lemma 3.5, we see that $x^{*}$ is a maximal extended Nash equilibrium and $x_{*}$ is a minimal extended Nash equilibrium of this game. This completes the proof of the theorem. 
Corollary 3.7 Under the condition (II) of Theorem 3.6, if every player's strategy set $\left(S_{i}, \preceq_{i}\right)$ is a strongly inductive poset for $i \in N$ and his payoff function $P_{i}$ furthermore satisfies

(III) for any fixed $i \in N$ and $x_{-i} \in S_{-i}, P_{i}\left(S_{i}, x_{-i}\right)$ is an inversely inductive poset in $\left(U, \preceq^{U}\right)$, then the game $\Gamma$ has an extended Nash equilibrium. Furthermore, $\Gamma$ has minimal and maximal extended Nash equilibriums.

Proof In order to check that all conditions of Theorem 3.6 are satisfied, it suffices to check that $S_{i}$ is inversely inductive for each $i \in N$. Let $C=\left\{x_{k}\right\}_{k=1}^{\infty}$ be an arbitrary c.c. of $S_{i}$. Then, for fixed $x_{-i} \in S_{-i}$, by the condition (II) $P_{i}\left(C, x_{-i}\right)$ is also a c.c. of $P_{i}\left(S_{i}, x_{-i}\right)$. By means of (III), there exists $y \in P_{i}\left(S_{i}, x_{-i}\right)$ such that $y \preceq^{U} P_{i}\left(x_{k}, x_{-i}\right)$ for each $k=1,2, \ldots$. We can choose $x \in S_{i}$ such that $y=P_{i}\left(x, x_{-i}\right)$. By means of the condition (II), we have $x \preceq_{i} x_{k}$ for $k=1,2, \ldots$, that is, $x$ is a lower bound of $C$. Consequently, $S_{i}$ is inversely inductive. Now Theorem 3.6 guarantees the desired results.

Remark 3.8 In addition to (II), all the rest of the hypotheses in Corollary 3.7, respectively, correspond to the conditions (i) and (2) in [6], Theorem 3.4. However, we not only use the weaker inductive poset concept but also reduce some tedious hypotheses.

\section{Example}

The purpose of this section is to show the advantages of our results by the following example.

Example 4.1 (Military manufacture example) Suppose that a war is kindling between two countries $C 1$ and $C 2$. To strengthen the combat effectiveness, they both intend to invest funds to acquire more weapons. Suppose that there are two military factories $F 1$ and $F 2$ offering the weapons to the two countries $C 1$ and $C 2$, respectively. As there is shortage of funds, each country can only invest 80 million dollars into its military factory. Suppose that the two factories just produce two weapons of the same type, tankers and fighter planes. The manufacturing cost of a tanker is 20 million dollars and a fighter plane will cost 30 million dollars. Suppose that the incomes are determined by the number of the two weapons that the factory can make. An arbitrarily considered outcome is a set of the total combat effectiveness of a factory. Let $U$ be the collection of all possible outcomes. Assume that the combat effectiveness of a tanker and a fighter plane is incomparable. It is obvious that $\left(U, \preceq^{U}\right)$ is a poset.

From what has been described above, we easily get the feasible strategy set of $\mathrm{Fi}$ as $S_{i}=\left\{A_{1}=(4,0), A_{2}=(3,0), A_{3}=(2,1), A_{4}=(1,2), A_{5}=(0,2)\right\}$, where the first and second components of $A_{k}$ with $k \in\{1,2, \ldots, 5\}$ denote the number of tankers and the number of fighter planes produced by Fi, respectively. Let $P_{i}: S \rightarrow U$ be the payoff function of Fi, where, for any $x \in S, P_{i}(x)$ denotes the total combat effectiveness of the weapons produced by Fi. We are now in the position to find an extended Nash equilibrium of this NNG.

Proof For any $A_{k}, A_{t} \in S_{i}$ with $A_{k}=\left(a_{1}, a_{2}\right)$ and $A_{t}=\left(b_{1}, b_{2}\right)$, we define the partial order $\preceq_{i}$ on $S_{i}$ as follows:

$$
A_{k} \preceq_{i} A_{t} \quad \text { if and only if } \quad a_{i} \leq b_{i}, \quad \text { for any } k, t \in\{1,2, \ldots, 5\} \text { and } i \in\{1,2\} .
$$


Obviously, $\left(S_{i}, \preceq_{i}\right)$ is a poset. Let $S=S_{1} \times S_{2}$. For any $x, y \in S$ with $x=\left(A_{k}, A_{t}\right)$ and $y=$ $\left(A_{m}, A_{n}\right), k, t, m, n \in\{1,2, \ldots, 5\}$, the partial order $\preceq^{S}$ on $S$ is induced by the partial order $\preceq_{i}$ as follows:

$$
x \preceq^{S} y \quad \text { if and only if } \quad A_{k} \preceq_{1} A_{m} \text { and } A_{t} \preceq_{2} A_{n}, \quad \text { for any } k, t, m, n \in\{1,2, \ldots, 5\} .
$$

Then $\left(S, \preceq^{S}\right)$ is a poset.

It is obvious that the strategy set $\left(S_{i}, \preceq_{i}\right)$ is a strongly inductive and inversely inductive poset. Therefore the condition (I) of Theorem 3.6 is satisfied. For any two strategies $x=$ $\left(x_{1}, x_{2}\right), y=\left(y_{1}, y_{2}\right) \in S$ with $x \preceq^{S} y$, we have $x_{i} \preceq_{i} y_{i}$ for each $i \in\{1,2\}$, which means that for each factory, both the number of tankers and the number of fighter planes produced in $x$ are less than that in $y$. Hence, the total combat effectiveness of the weapons in $x$ is weaker than that in $y$ for each factory, that is, $P_{i}(x) \preceq^{U} P_{i}(y)$ for $i \in\{1,2\}$. Then the sufficiency of (II) in Theorem 3.6 is satisfied. We now show that the game also meets the necessity of (II). For any given $P_{i}(x), P_{i}(y) \in U$ with $P_{i}(x) \preceq^{U} P_{i}(y), i \in\{1,2\}$, we are in the position to prove that $x \preceq^{S} y$. If $x$ and $y$ are incomparable, then the numbers of tankers and fighter planes in $x$ will not simultaneously be less or more than that in $y$. This implies that neither $P_{i}(x) \preceq^{U} P_{i}(y)$ nor $P_{i}(y) \preceq^{U} P_{i}(x)$ since the combat effectiveness of a tanker and a fighter plane is incomparable. Therefore, $x$ and $y$ are comparable by hypothesis $P_{i}(x) \preceq^{U} P_{i}(y)$, that is, $x \preceq^{S} y$ or $y \preceq^{S} x$. By the sufficiency of (II), if $y \preceq^{S} x$, then $P_{i}(y) \preceq^{U} P_{i}(x)$, which is a contradiction. Consequently, $x \preceq^{S} y$. By virtue of Theorem 3.6, the game has maximal and minimal extended Nash equilibriums. In fact, it easily checks that the strategy $\left(A_{1}, A_{1}\right) \in$ $S$ is not only a maximal extended Nash equilibrium but also a minimal extended Nash equilibrium of this game.

Remark 4.2 It is easy to see that $\left(S_{i}, \preceq_{i}\right)$ in Example 4.1 has neither a sup-center nor an inf-center, which indicates that Theorem 3.4 of [6] cannot solve the problem above. Hence our theorem compares favorably with that of [6] in its particular way.

Competing interests

The authors declare that they have no competing interests.

Authors' contributions

All authors contributed equally to this work. All authors read and approved the final manuscript.

\section{Acknowledgements}

The article was supported by Natural Science Foundation of China (71471051) and Natural Science Foundation of Zhejiang Province (LY12A01002).

Received: 17 December 2014 Accepted: 21 April 2015 Published online: 06 May 2015

\section{References}

1. Facchinei, F, Kanzow, C: Generalized Nash equilibrium problems. Ann. Oper. Res. 175, 177-211 (2010). doi:10.1007/s10479-009-0653-x

2. Carl, S, Heikkilä, S: Fixed Point Theory in Ordered Sets and Applications: From Differential and Integral Equations to Game Theory. Springer, New York (2010)

3. Li, JL: Several extensions of the Abian-Brown fixed theorem and their applications to extended and generalized Nash equilibria on chain-complete posets. J. Math. Anal. Appl. 409, 1084-1092 (2014)

4. Li, JL: Applications of fixed point theory to generalized Nash equilibriums of nonmonetized noncooperative games on Banach lattices. Nonlinear Anal. Forum 18, 1-11 (2013)

5. Li, JL, Park, S: Generalized Nash equilibria of nonmonetized noncooperative games on lattices. Br. J. Econ. Manag. Trade 4(1), 97-110 (2014)

6. Xie, LS, Li, JL, Wen, CF: Applications of fixed point theory to extended Nash equilibriums of nonmonetized noncooperative games on posets. Fixed Point Theory Appl. 2013, 235 (2013)

7. Xie, LS, Li, JL, Yang, WS: Order-clustered fixed point theorems on chain-complete preordered sets and their applications to extended and generalized Nash equilibria. Fixed Point Theory Appl. 2013, 192 (2013) 
8. Agarwal, R, Balaj, M, O'Regan, D: A unifying approach to variational relation problems. J. Optim. Theory Appl. 155, 417-429 (2012). doi:10.1007/s10957-012-0090-x

9. Aliprantis, CD, Burkinshaw, O: Positive Operators. Springer, Berlin (2006)

10. Dunford, N, Schwartz, JT: Linear Operators. Part I: General Theory. Wiley-Interscience, New York (1988)

11. Hong, SH, Qiu, ZY: Fixed points of discontinuous multivalued operators in ordered spaces with applications. Fixed Point Theory Appl. 2010, 745769 (2010). doi:10.1155/2010/745769

Submit your manuscript to a SpringerOpen ${ }^{\circ}$ journal and benefit from:

- Convenient online submission

Rigorous peer review

- Immediate publication on acceptance

- Open access: articles freely available online

- High visibility within the field

- Retaining the copyright to your article 